\title{
EFECTO DE LA CORRIENTE EN CHORRO DE BAJOS NIVELES EN LA OCURRENCIA DE PRECIPITACIÓN EN LA SELVA DEL PERÚ
}

\section{EFFECT OF THE LOW-LEVEL JET STREAM ON THE OCCURRENCE OF PRECIPITATION IN THE PERUVIAN JUNGLE}

\author{
Jose Carlos Coello Fababa ${ }^{1}$ y Victoria Calle Montes $^{2}$
}

\begin{abstract}
Resumen
Se analizó la corriente en chorro de América del Sur (SALLJ, siglas en inglés) y la ocurrencia de precipitación sobre la selva del Perú, tomando en cuenta los datos del modelo atmosférico Global Forecast System (GFS) y datos de precipitación acumulada estimado por el satélite Tropical Rainfall Measuring Mission (TRMM) en los veranos australes comprendidos entre los años 2005 y 2014. Se utilizó la distribución de Weibull para el análisis estadístico del viento meridional del norte y el test estadístico no paramétrico de correlación de Kendall para asociar los eventos SALLJ definidos por los criterios de Whiteman et al. (1997) y Bonner (1968). Los resultados revelan que el comportamiento promedio de la componente meridional del viento fluctúa entre 1.2 y $11.7 \mathrm{~m} / \mathrm{s}$ con variaciones de $+/-3.2 \mathrm{~m} / \mathrm{s}$, registrando un viento máximo de $21.4 \mathrm{~m} / \mathrm{s}$. De un total de 39 casos, el $53.8 \%$ se identificó con las condiciones propuestas por Whiteman y un $46.2 \%$ con las condiciones de Bonner. Se registró una precipitación máxima de $64.00 \mathrm{~mm} /$ día y mayor número de días con precipitaciones asociadas a eventos SALLJ para las 00 UTC.
\end{abstract}

Palabras clave: corriente en chorro de América del Sur (SALLJ), distribución Weibull, cizalladura vertical.

\begin{abstract}
The South American jet stream (SALLJ) and the precipitation occurrence on the Peruvian jungle were analyzed, taking into account data from the Global Forecast System (GFS) atmospheric model and accumulated precipitation data estimated by the Tropical Rainfall Measuring Mission (TRMM) satellite in the southern summers between the years 2005 and 2014. The Weibull distribution was used for the statistical analysis of the southern north wind and the Kendall's non-parametric correlation statistical test to associate the SALLJ events defined by the criteria of Whiteman et al. (1997) and Bonner (1968). The results reveal that the average behavior of the southern component of the wind fluctuates between 1.2 and $11.7 \mathrm{~m} / \mathrm{s}$ with variations of $+/-3.2 \mathrm{~m} / \mathrm{s}$, registering a maximum wind of $21.4 \mathrm{~m} / \mathrm{s}$. Of a total of 39 cases, $53.8 \%$ were identified with the conditions proposed by Whiteman and $46.2 \%$ with the conditions of Bonner. Maximum rainfall of 64.00 $\mathrm{mm} /$ day and a greater number of days with rainfall associated with SALLJ events were recorded for 00 UTC.
\end{abstract}

Key words: South American jet stream (SALLJ), Weibull distribution, vertical shear.

\section{Introducción}

La Corriente en Chorro de bajos niveles en América del Sur (South American Low Level Jet - SALLJ; siglas en inglés), es un flujo de viento meridional $\left(\overline{\mathrm{V}}_{\mathrm{m}}\right.$, a partir de ahora) entre los 850 y $700 \mathrm{hPa}$ a 1.5 y $3 \mathrm{~km}$ de altura, respectivamente; presente durante el año. Se intensifica en el verano austral debido al gradiente térmico diurno y la mayor incidencia de radiación solar, que permite el desarrollo de sistemas dinámicos en la atmosfera. Este flujo de viento meridional del norte caracterizado por velocidades mayores o iguales a $12 \mathrm{~m} / \mathrm{s}$ según el criterio 1 (Bonner, 1968) o $10 \mathrm{~m} / \mathrm{s}$ según el criterio 0 (Whiteman et al., 1997), recorre por los países de Venezuela, Colombia, Perú, Bolivia, Argentina y Paraguay transportando la humedad del Océano Atlántico central y de la cuenca del Amazonas, produciendo una advección de aire cálido y húmedo canalizado por el lado Este de la Cordillera de los Andes en dirección al Sur (Marengo et al., 2001).

El SALLJ en su recorrido modula el tiempo atmosférico y clima en la selva amazónica, influyendo en el transporte de humedad, distribución de precipitación, temperatura, nubosidad, entre otros (Amador, 2008). Además, puede alterar los periodos secos y lluviosos dependiendo de la cantidad de vapor de agua disponible, cambiando la temperatura de una región e incrementar o disminuir el nivel de agua en una especie por transpiración o evaporación en los bosques (Jactel et al., 2017).

Diferentes trabajos realizados con datos de reanálisis NCEP-NCAR (Marengo et al., 2004), ERA (Salio et al., 2002) y ETA/CPTEC (Saulo et al., 2000) concluyeron que la mayor frecuencia del SALLJ se da durante el verano austral, entre las 06 - 12 UTC (12 UTC principalmente), y en el invierno austral, entre las 
00 - 06 UTC (06 UTC principalmente). La humedad transportada contribuye a la formación de complejos convectivos y la liberación del calor latente sobre la cuenca del Río de la Plata (salida del SALLJ). Los eventos SALLJ que influyen sobre Perú mayormente ocurren en la estación del verano austral en niveles cercanos a $850 \mathrm{hPa}$, donde se presenta una atmosfera baroclínica durante la presencia del SALLJ (Marengo et al., 2001).

El mecanismo físico para el desarrollo del SALLJ ocurre por la interacción entre la radiación solar, la tierra y el efecto topográfico de la Cordillera de los Andes, cuyo origen parte del centro del Mar del Caribe (Torrealba \& Amador, 2010). Las variaciones más notorias se dan al comenzar la noche y al comenzar la madrugada con las velocidades más altas. La circulación presenta una variación diurna y los principales mecanismos son: el gradiente de temperatura horizontal causado por el diferente tipo de cobertura superficial (suelo con vegetación y suelo desnudo), efecto de fricción y presencia de un viento geostrófico hacia el sur (Wu \& Raman, 1997).

En 1995, 1998, 2005 y 2010 se registraron las sequias más extremas en la cuenca del río Amazonas; entre ellas, la del año 2010 fue una particularmente severa, comprendida entre los meses de abril y agosto, con un debilitamiento de los vientos alisios (moduladores de eventos SALLJ) y una precipitación mayor en la zona del Océano Atlántico central, que provocó un menor transporte de vapor de agua (disminuyendo hasta en un 56\%) en los flujos de viento hacia el lado occidental del Amazonas y una anomalía positiva en la temperatura superficial del mar sobre el Océano Atlántico norte, favoreciendo la liberación del calor latente sobre el Océano Atlántico (Espinoza et al., 2011).

Se realizó un estudio utilizando datos de radiosondeo tomados del experimento DESMATA (Impacto de la deforestación a lo largo de la costa atlántica de la Amazonía) y CIMELA (Circulaciones de mesoescala en la Amazonia oriental) en la localidad de Ajuruteua $\left(00^{\circ} 50^{\prime} \mathrm{S}, 46^{\circ} 38^{\prime} \mathrm{W}\right.$, Brasil) del 8 al 22 de abril del 2002 durante el periodo lluvioso y del 27 octubre al 15 noviembre del 2003 en el periodo seco con la finalidad de registrar eventos SALLJ en estaciones diferentes del año; de los resultados obtenidos, durante el periodo seco, se registraron 3 veces más que en el periodo lluvioso (Sousa et al., 2006). Además, se comprobó que en fases cálidas del ENSO, los SALLJ son más intensos que en periodos fríos del ENSO (Vernekar et al., 2003).

El objetivo de este trabajo fue conocer la relación entre la precipitación superior a los $0.1 \mathrm{~mm} /$ día asociada a eventos SALLJ. Para ello, el presente estudio buscó conocer el comportamiento y condiciones del SALLJ por medio de mecanismos físicos en el transporte de la humedad por el lado Este de la Cordillera de los Andes sobre la selva del Perú durante el verano austral entre los años 2005 y 2014.

\section{Materiales y métodos}

\section{Área de estudio}

El área de estudio (Figura 1) se definió en función a la elevación de la superficie que comprende las regiones naturales Yunga fluvial (500 - $2300 \mathrm{msnm})$, Rupa Rupa (500 - 1500 msnm) y Omagua (debajo de $500 \mathrm{msnm}$ ), que agrupan diferentes características en cuanto a topografía y condiciones de tiempo y clima. Ubicadas al lado Este de los Andes peruanos entre los $0.1^{\circ} \mathrm{S}-14.4^{\circ} \mathrm{S}$ y $78.8^{\circ} \mathrm{W}-68.8^{\circ} \mathrm{W}$, la topografía de la zona es muy variada debido a la presencia de la Cordillera de los Andes.

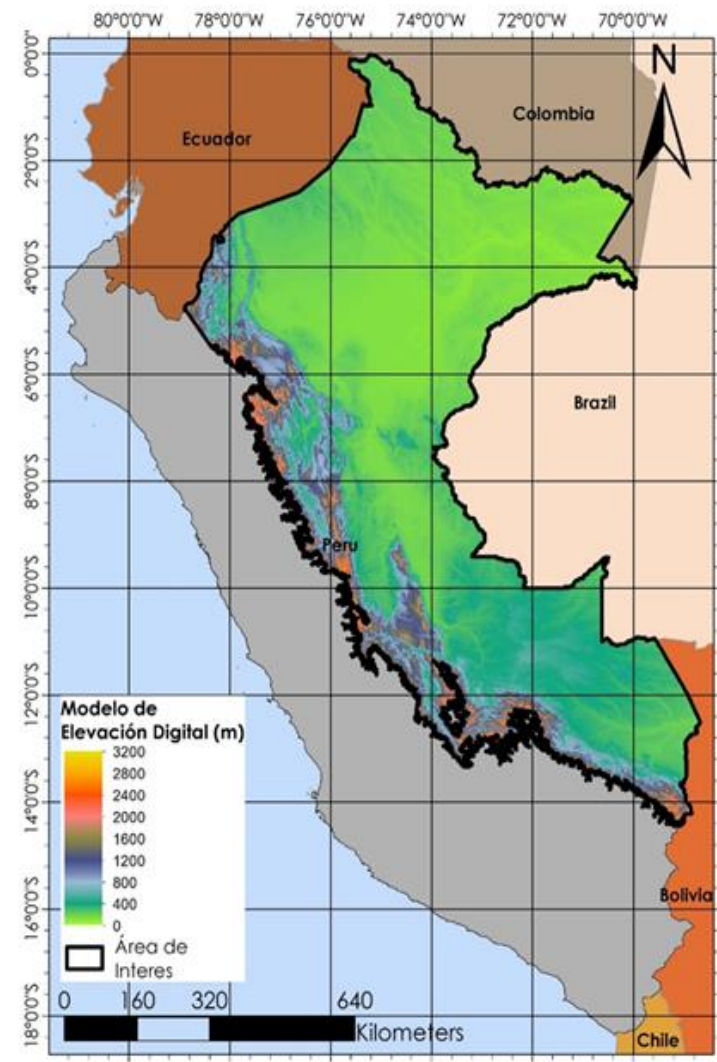

Figura 1. Área de estudio.

Los datos fueron obtenidos del modelo GFS (Global Forecast System) a una resolución espacial de $2.5^{\circ}$ en las horas sinópticas 00, 06, 12 y 18 UTC para los meses de diciembre, enero y febrero (verano austral, a partir de ahora), con un $21.5 \%$ de datos faltantes (archivo de descripción de datos ausente en la fuente de descarga). Los datos de precipitación diaria fueron analizados de la misión de medición de las precipitaciones tropicales (TRMM, de aquí en adelante) del producto 3B42V7 a $0.25^{\circ}$ (Yupanqui et al., 2015) debido a su mayor precisión y calidad de los datos (Huffman et al., 2007). En la relación de estas variables se ajustó al tamaño de pixel a $0.1^{\circ}$ y fueron analizadas en el periodo 2005 al 2014. 
Identificación de eventos SALLJ

Se consideraron los criterios propuestos por Bonner (1968) al estudiar la frecuencia e intensidad de los flujos de viento sobre los EE.UU. con un registro de perfiles de viento durante 2 años en 47 estaciones de radiosondeo, y el propuesto por Whiteman et al. (1997) que, al identificar flujos de viento que no cumplían los criterios propuestos por Bonner, incluyó un criterio más en su estudio, el cual considera vientos menos intensos. Por ello, se consideró en este estudio como criterio cero y criterio uno a Whiteman et al. (1997) y Bonner (1968), respectivamente, cuyas características se detallan a continuación:

- Criterio cero, considera el $\bar{V}_{m}$ sobre el nivel isobárico de $850 \mathrm{hPa}$ igual o superior a $10 \mathrm{~m} / \mathrm{s}$ y una cizalladura vertical de por lo menos $5 \mathrm{~m} / \mathrm{s}$ entre $10 \mathrm{~s} 850$ y $700 \mathrm{hPa}$, que debe ubicarse dentro del área de la isotaca de $10 \mathrm{~m} / \mathrm{s}$.

- Criterio uno, considera el $\bar{V}_{\mathrm{m}}$ sobre el nivel isobárico de $850 \mathrm{hPa}$ igual o superior a $12 \mathrm{~m} / \mathrm{s}$ y una cizalladura vertical de por lo menos $6 \mathrm{~m} / \mathrm{s}$ entre los 850 y $700 \mathrm{hPa}$, que debe ubicarse dentro del área de la isotaca de $12 \mathrm{~m} / \mathrm{s}$.

Ambos criterios deben ser analizados al lado Este de la Cordillera de los Andes.

La cizalladura vertical ( $\left.\mathrm{V}_{\text {ciz.vert }}\right)$, para las 00, 06, 12 y 18 UTC del $\bar{V}_{\mathrm{m}}$, fue calculada por la Fórmula 1.

$$
\mathrm{V}_{\text {ciz.vert }}=\mathrm{V}_{\max (850 \mathrm{hPa})}-\mathrm{V}_{3 \mathrm{~km}(700 \mathrm{hPa})}
$$

Fórmula 1. Cálculo de la cizalladura vertical

$$
\left(\mathrm{V}_{\text {ciz.vert }}\right) \text {. }
$$

Donde el $\bar{V}_{m}$ máximo a $850 \mathrm{hPa} \mathrm{V}_{\max (850 \mathrm{hPa})}$ y el viento $\mathrm{V}_{3 \mathrm{~km}(700 \mathrm{hPa})}$ representan el viento meridional del norte a un nivel isobárico de $850 \mathrm{hPa}$ y $700 \mathrm{hPa}$, respectivamente. Se presentan tres situaciones con: A) $\mathrm{V}_{\max (850 \mathrm{hPa})}>\mathrm{V}_{3 \mathrm{~km}(700 \mathrm{hPa})}$ con valores de $\mathrm{V}_{\text {ciz.vert }}<0$, que muestran mejores condiciones para los eventos SALLJ; B) $\mathrm{V}_{\max (850 \mathrm{hPa})}<\mathrm{V}_{3 \mathrm{~km}(700 \mathrm{hPa})}$ con valores de $\mathrm{V}_{\text {ciz.vert }}>0$, que bloquean la formación de un evento SALLJ; y finalmente C) $V_{\max (850 \mathrm{hPa})}=V_{3 \mathrm{~km}(700 \mathrm{hPa})}$ con $\mathrm{V}_{\text {ciz.vert }}=0$, que no genera cortantes de viento.

Análisis temporal del viento

Se utilizó la distribución de Weibull debido a la sencillez y capacidad de ajustar la función de distribución de probabilidad para los datos de viento (Celik, 2003). Planteada en un principio por Weibull (1951), es considerada una distribución de probabilidad continua y multiparamétrica que viene siendo utilizada muy ampliamente en el análisis de la velocidad del viento. La función de distribución de probabilidad se muestra en la Fórmula 2.

$$
\begin{gathered}
f_{(v)}=\left(\frac{k}{c}\right)\left(\frac{v-T_{0}}{c}\right)^{k-1} e^{-\left(\frac{v_{i}-T_{0}}{c}\right)^{k}} \\
p_{i\left(v \leq v_{i}\right)}=1-e^{-\left(\frac{v_{i}-T_{0}}{c}\right)^{k}}
\end{gathered}
$$

Fórmula 2. Función de distribución de probabilidad.

Donde el valor de la velocidad del viento " $\mathrm{v}_{\mathrm{i}}$ " en un determinado momento "i", la velocidad promedio representada por el parámetro de escala "c" en m/s, el grado de dispersión de los datos por el parámetro de forma "k", y " $\mathrm{T}_{0}$ " parámetro de localización (parámetro que localiza la abscisa a partir de la cual se inicia la distribución); estos parámetros, reemplazados en la ecuación, muestran la frecuencia de la velocidad " $v$ " del viento (Silva et al., 2002) y " $\mathrm{p}_{\mathrm{i}}$ " representa la probabilidad de ocurrencia de la velocidad del viento a estimar.

Para la estimación de los parámetros de Weibull se utilizó el método de mínimos cuadrados estudiado por Touré (2005), el cual permite calcular los parámetros de forma "k" y escala "c" mediante la transformación doble logarítmica (Figura 2) aplicada a la función de distribución de probabilidad acumulada (CDF) de Weibull $\left(\mathrm{p}_{\mathrm{i}\left(\mathrm{v} \leq \mathrm{v}_{\mathrm{i}}\right)}\right)$, permitiendo transformar la CDF en una ecuación lineal de regresión.



Figura 2. Recta de ajuste usando el Método de Mínimos Cuadrados.

En este estudio se considera una distribución biparamétrica (Figura 3); es decir, está definida solo por el cálculo de los parámetros de forma y escala, ya que el tercer parámetro hace referencia a la variable tiempo, o conocido también como el parámetro de localización $\left(\mathrm{T}_{0}=0\right)$ de inicio de la función, que permite ingresar una perturbación (identificar fallas) a las condiciones iniciales al momento de generar la función de distribución de probabilidad de Weibul $\left(\mathrm{f}_{\mathrm{v}}\right)$. 


\section{Relación entre eventos SALLJ y precipitación}

El grado de asociación entre la precipitación y el $\overline{\mathrm{V}}_{\mathrm{m}}$ fue determinado mediante el coeficiente de Kendall. Este es un método robusto que no depende del tipo de distribución de datos y es resistente al no ser afectado por los datos atípicos (Wilks, 2006). Para calcular el estadístico de tamaño " $n$ " con datos parados, se tiene "X" e "Y", donde se utiliza la Fórmula 3 (Siegel \& Castellan, 1995).

$$
\begin{gathered}
\mathrm{T}=\frac{2 \mathrm{~S}}{\sqrt{\mathrm{n}(\mathrm{n}-1)-\mathrm{T}_{\mathrm{X}}} \sqrt{\mathrm{n}(\mathrm{n}-1)-\mathrm{T}_{\mathrm{y}}}} \\
\mathrm{S}=\mathrm{P}-\mathrm{M} \\
\mathrm{T}_{\mathrm{X}}=\sum \mathrm{t}(\mathrm{t}-1) \\
\mathrm{T}_{\mathrm{y}}=\sum \mathrm{t}(\mathrm{t}-1)
\end{gathered}
$$

\section{Fórmula 3.}

Donde "P" representa el número de valores positivos (acuerdos) asociado al número de veces de incrementos de "Y" conforme incrementa "X", y "M" representa el número de valores negativos (desacuerdos) asociado al número de veces que disminuye "Y" conforme incrementa "X". " $\mathrm{T}_{\mathrm{x}}$ ", y " $\mathrm{T}_{\mathrm{y}}$ " representa el número de observaciones " $\mathrm{t}$ " empatadas para cada grupo de empates de la variable "X" e "Y", respectivamente. De esta manera, los datos de precipitación diaria acumulada del TRMM y el " $\overline{\mathrm{V}}_{\mathrm{m}}$ " fueron asociados utilizando el coeficiente de correlación de Kendall para las 4 horas sinópticas. Luego, el grado de relación entre el " $\overline{\mathrm{V}}_{\mathrm{m}}$ " y la precipitación diaria acumulada, fueron superpuestas sobre las zonas de eventos SALLJ.

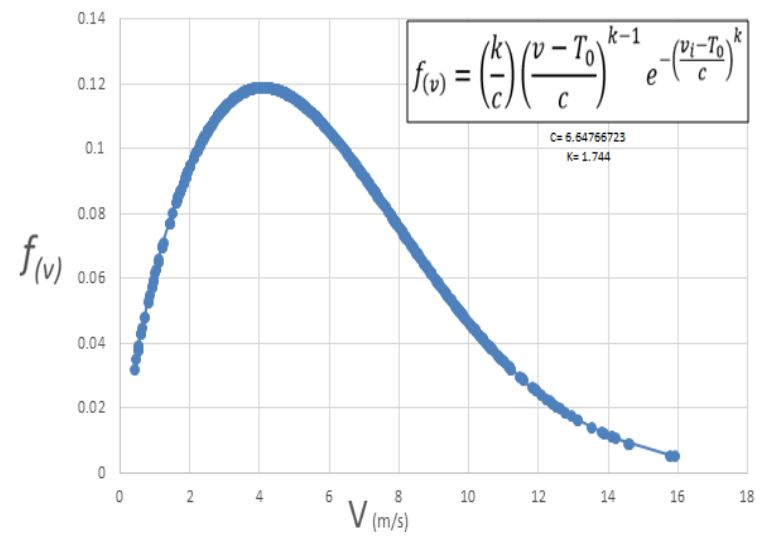

Figura 3. Distribución de Weibull para un factor de forma y escala fijas.

\section{Resultados y discusión}

Comportamiento del SALLJ durante el verano austral entre el $2005 \mathrm{al} 2014$

Se identificó cuantitativamente (Figura 4) una máxima correlación entre $\bar{V}_{\mathrm{m}}$ y la $\mathrm{V}_{\text {ciz.vert }}$ a las $00 \mathrm{UTC}$ (19 hora local en Perú) sobre el lado Noreste entre los $2.2^{\circ} \mathrm{S}-6.4^{\circ} \mathrm{S}$ y $75.6^{\circ} \mathrm{W}-70^{\circ} \mathrm{W}$ correspondiente a la zona 1 (a partir de ahora) con correlaciones entre 0.36 y 0.5. Por el lado Este de la cordillera central de los Andes, entre los $10.4^{\circ} \mathrm{S}-12.42^{\circ} \mathrm{S}$ y $75.6^{\circ} \mathrm{W}-72.4^{\circ} \mathrm{W}$ correspondientes a la zona 2 (a partir de ahora), se obtuvieron correlaciones de 0.2 a 0.5 y al Sureste entre los $12.42^{\circ} \mathrm{S}-14.4^{\circ} \mathrm{S}$ y $71.6^{\circ} \mathrm{W}-68.8^{\circ} \mathrm{W}$ para la zona 3 (a partir de ahora) se muestran correlaciones de 0.1 a 0.4; se utilizó las 00 UTC como base para realizar la zonificación debido a que presenta los valores máximos asociados a una relación entre el $\overline{\mathrm{V}}_{\mathrm{m}}$ y $\mathrm{V}_{\text {ciz.vert }}$, similar a los resultados obtenidos en el estudio de Marengo et al. (2004). Se considera una correlación baja de $-0.3 \leftrightarrow 0.3$ y correlación media de $0.3 \leftrightarrow 0.5$. Además, se observó en horas de la noche 00 UTC sobre la zona 1 y 2 , el máximo grado de asociación entre $\bar{V}_{\mathrm{m}}$ y $\mathrm{V}_{\text {ciz.vert. Es }}$ decir, la componente del viento meridional del norte a $850 \mathrm{hPa}$ es superior al viento a $700 \mathrm{hPa}$. Sin embargo, en la zona 3 no se evidenció un grado de asociación marcado. La influencia de la topografía en la zona 2 y 3 juega un rol muy importante que afecta la componente del viento meridional. De la misma forma, para las 06, 12 y 18 UTC (01, 07 y 13 hora local, respectivamente), la correlación entre las variables de $\overline{\mathrm{V}}_{\mathrm{m}}$ y $\mathrm{V}_{\text {ciz.vert }}$ registró los valores más bajos, lo que responde a la naturaleza de formación del SALLJ que parte de un intercambio energético con el entorno.

Se registró un $\bar{V}_{m}$ promedio máximo a $850 \mathrm{hPa}$ entre $\operatorname{los} 9.3$ a $10.5 \mathrm{~m} / \mathrm{s}$ a las 12 UTC sobre la zona 1 , seguido por las 06 UTC registrando valores entre los 5.8 a 8.2 $\mathrm{m} / \mathrm{s}$. En cambio, los vientos menos intensos (inferior a $5.8 \mathrm{~m} / \mathrm{s}$ ) al lado Este de la Cordillera de los Andes (zona 2 y 3 ), muestran el efecto topográfico, donde el efecto de la fricción debilita y altera los flujos de vientos que son canalizados hacia el sur (Figura 5). De acuerdo al comportamiento temporal de la $\mathrm{V}_{\text {ciz.vert, la mayor }}$ inestabilidad se ubicó en la zona 2 para las 12 UTC con valores superiores a los $4.1 \mathrm{~m} / \mathrm{s}$. Sobre los terrenos planos (zona 1), el efecto de la cortante vertical muestra valores intermedios en la componente meridional del viento con dirección al sur cercano a los $2 \mathrm{~m} / \mathrm{s}$ y valores muy bajos entre 0.5 y $2 \mathrm{~m} / \mathrm{s}$ (Figura 6).

Asimismo, los valores máximos registrados (Figura 7), en la serie temporal de datos durante el verano austral comprendido entre los años 2005 y 2014, evidencian las máximas velocidades sobre el Noreste del territorio peruano, con valores de 14.6 a $21.4 \mathrm{~m} / \mathrm{s}$ a las 12 UTC sobre la zona 1, seguido de las 06 UTC con valores de 12.8 a $14.6 \mathrm{~m} / \mathrm{s}$; siendo las 00 y 18 UTC las horas sinópticas que registraron los valores entre los 10.4 y $13.7 \mathrm{~m} / \mathrm{s}$ en la componente meridional del norte; estos últimos presentaron los valores más bajos en la 
intensidad del viento máximo registrado sobre la zona 1. En el caso de las zonas 2 y 3, las intensidades oscilaron entre los 1.8 y $12.8 \mathrm{~m} / \mathrm{s}$, para las 00,06 y 18 UTC. Así mismo, las intensidades de los vientos más bajos se registraron cerca de las laderas de la Cordillera de los Andes (lado Este), caso contrario sucedió a medida que nos alejamos de la cordillera (en dirección hacia la selva).

Sobre el factor de forma (Figura 8), que indica el grado de dispersión de la velocidad del viento respecto a su valor medio, los valores más bajos representan una menor variación en la velocidad del viento promedio y una mayor frecuencia del viento promedio. De manera particular, esto sucede para la zona 2 y 3, cercanas a las laderas de la Cordillera de los Andes para las 00, 06, y 12 UTC con valores que oscilan entre 0 y $1.4 \mathrm{~m} / \mathrm{s}$. Las mayores variaciones en el $\overline{\mathrm{V}}_{\mathrm{m}}$ se muestran en la zona 1 , donde supera en $2.3 \mathrm{~m} / \mathrm{s}$ del promedio normal; de tal manera que la influencia de los vientos alisios se hace más predominante debido a la deflexión hacia el sur que se genera por la topografía.

El número de casos registrados para cada año muestra una relación similar comparada con la precipitación acumulada en los eventos SALLJ. El año con mayor registro de eventos se dio en el 2007, con 13 casos, seguido del 2008, 2006, 2012, 2009, 2010, 2011 y por último 2005 con $9,5,4,3,2,2$ y 1 casos, respectivamente.

Aparte de ello, los eventos SALLJ durante las horas sinópticas analizadas evidencian un mayor número de casos a las 00 y 12 UTC correspondientes al $76.9 \%$ de los casos identificados, durante las horas intermedias (06 UTC) se registró un $17.9 \%$ y finalmente un $5.1 \%$ del total de casos correspondiente a las 18 UTC. Analizando este factor y su equivalente a hora local, se encontraría con mayor probabilidad eventos SALLJ entre las 19:00 y 07:00 horas, asociados al anochecer y amanecer.

De los criterios analizados para la identificación de los eventos SALLJ, se registró un $53.8 \%$ de los casos utilizando el criterio " 0 " propuesto por Whiteman et al. (1997) y un 46.2\% utilizando el criterio " 1 " propuesto por Bonner (1968). Se observó una mayor frecuencia de eventos SALLJ sobre la zona 1 (76.9\% de casos), seguida de la zona 3 (23.1\% de casos). Cabe mencionar que, no se evidenciaron las condiciones necesarias para identificar un SALLJ sobre la zona 2. También se registró $64.1 \%$ de eventos SALLJ en el mes de febrero y un $35.9 \%$ para los meses de diciembre y enero.

Precipitación diaria acumulada y eventos SALLJ sobre la selva del Perú en el verano austral entre los años 2005 al 2014

Se analizó el comportamiento temporal (Figura 9, muestra un caso de un total de 12 gráficas) de la intensidad y cizalladura del viento meridional del norte con la precipitación diaria acumulada sobre los pixeles centrales de las 3 zonas identificadas en la Figura 4. Se considera la cizalladura menor a $-5 \mathrm{~m} / \mathrm{s}$ y una intensidad de viento inferior a $-10 \mathrm{~m} / \mathrm{s}$; los valores negativos corresponden a la orientación de la componente meridional del Norte (con dirección al Sur) y las precipitaciones superiores a los $0.1 \mathrm{~mm} /$ día.

Los días que cumplen los criterios para un evento SALLJ (Tabla 1) no necesariamente están asociados a fuertes precipitaciones. De los 39 casos identificados, el $28.2 \%$ de eventos SALLJ registraron, según los datos de precipitación acumulada, valores entre 0 a 0.2 $\mathrm{mm} /$ día, un $56.4 \%$ de casos con precipitaciones entre los 0.6 y $8.9 \mathrm{~mm} /$ día, y finalmente un $15.4 \%$ de casos registraron precipitaciones acumuladas entre 13 y 64 $\mathrm{mm} /$ día.

Tabla 1. Eventos SALLJ identificados en el periodo de estudio.

\begin{tabular}{|c|c|c|c|c|c|c|c|}
\hline & Fecha & & 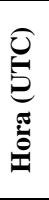 & 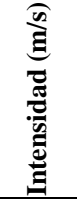 & 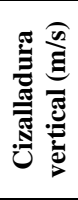 & 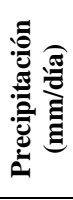 & $\stackrel{\Xi}{\tilde{N}}$ \\
\hline 2005 & JAN & 20 & 18 & -10.3 & -5.4 & 6.0 & 1 \\
\hline \multirow[t]{5}{*}{2006} & FEB & 7 & 12 & -10.8 & -5.4 & 2.2 & 1 \\
\hline & & 8 & 12 & -12.1 & -6.9 & 3.2 & 1 \\
\hline & & 14 & 12 & -15.4 & -9.3 & 2.8 & 1 \\
\hline & & 22 & 12 & -13.5 & -5.9 & 0.0 & 1 \\
\hline & $\mathrm{DEC}$ & 9 & 0 & -12.3 & -10.0 & 0.6 & 1 \\
\hline \multirow[t]{13}{*}{2007} & JAN & 8 & 6 & -13.1 & -6.3 & 6.6 & 1 \\
\hline & & 31 & 0 & -12.8 & -6.7 & 1.4 & 1 \\
\hline & FEB & 4 & 12 & -10.1 & -5.2 & 8.9 & 1 \\
\hline & & 6 & 6 & -10.8 & -5.6 & 0.0 & 1 \\
\hline & & 7 & 6 & -13.2 & -7.5 & 0.1 & 1 \\
\hline & & 7 & 12 & -12.1 & -7.5 & 0.1 & 1 \\
\hline & & 15 & 12 & -12.8 & -5.5 & 0.0 & 1 \\
\hline & & 16 & 12 & -13.2 & -6.3 & 0.0 & 3 \\
\hline & & 16 & 0 & -12.3 & -18.6 & 16.6 & 1 \\
\hline & & 17 & 0 & -11.5 & -5.6 & 17.5 & 3 \\
\hline & & 18 & 0 & -12.5 & -7.0 & 28.4 & 3 \\
\hline & & 20 & 0 & -10.1 & -5.5 & 1.7 & 1 \\
\hline & & 20 & 12 & -14.9 & -6.9 & 1.7 & 1 \\
\hline \multirow[t]{9}{*}{2008} & JAN & 3 & 12 & -13.5 & -6.2 & 0.0 & 1 \\
\hline & & 4 & 12 & -15.5 & -5.4 & 1.1 & 1 \\
\hline & & 25 & 0 & -15.0 & -6.5 & 1.2 & 1 \\
\hline & FEB & 1 & 6 & -12.2 & -5.5 & 0.0 & 1 \\
\hline & & 2 & 0 & -12.7 & -9.1 & 0.8 & 1 \\
\hline & & 10 & 6 & -13.6 & -6.3 & 43.3 & 3 \\
\hline & & 21 & 0 & -10.1 & -5.6 & 64.0 & 3 \\
\hline & & 24 & 0 & -10.3 & -5.1 & 4.7 & 1 \\
\hline & & 28 & 0 & -10.4 & -5.0 & 0.2 & 1 \\
\hline \multirow[t]{3}{*}{2009} & DEC & 6 & 12 & -10.4 & -5.9 & 13.0 & 3 \\
\hline & & 7 & 6 & -11.1 & -5.2 & 0.0 & 1 \\
\hline & & 29 & 0 & -12.8 & -8.3 & 3.6 & 3 \\
\hline \multirow[t]{2}{*}{2010} & $\mathrm{DEC}$ & 12 & 0 & -12.3 & -8.7 & 6.1 & 3 \\
\hline & & 28 & 12 & -10.1 & -5.6 & 4.2 & 1 \\
\hline \multirow[t]{2}{*}{2011} & FEB & 18 & 12 & -10.6 & -5.3 & 5.7 & 1 \\
\hline & $\mathrm{DEC}$ & 28 & 0 & -10.0 & -5.6 & 3.0 & 1 \\
\hline \multirow[t]{4}{*}{2012} & JAN & 31 & 0 & -10.8 & -5.3 & 4.4 & 3 \\
\hline & FEB & 3 & 12 & -12.7 & -7.0 & 5.4 & 1 \\
\hline & & 3 & 18 & -10.3 & -5.3 & 5.4 & 1 \\
\hline & & 24 & 6 & -11.7 & -5.7 & 0.1 & 1 \\
\hline
\end{tabular}

Los años con mayor precipitación acumulada durante los eventos SALLJ fueron en 2008, luego 2007, 2009, 2012, 2010, 2006, 2011 y finalmente 2005 con 115.2 , 82.8, 16.5, 15.2, 10.3, 8.9, 8.7 у $6 \mathrm{~mm}$, respectivamente. Dichos valores representan la suma 
de precipitación diaria durante los eventos SALLJ para cada año.

Por otro lado, un caso ilustrativo (1 caso de 39 identificados) muestra los cortes latitudinales (Figura 10) sobre los eventos SALLJ, se analizó el perfil del viento meridional $(\mathrm{m} / \mathrm{s}$, sombreado con colores), el omega $(\mathrm{Pa} / \mathrm{s}$, líneas punteadas de color negro), la humedad relativa (línea punteada de color azul con valores superiores al $70 \%$ ) y la temperatura potencial equivalente (en $\mathrm{K}$, línea punteada de color magenta) en diferentes niveles isobáricos (de $1000 \mathrm{hPa}$ a $100 \mathrm{hPa}$ ).

El comportamiento vertical de la atmósfera, visto desde un corte latitudinal a $2.9^{\circ} \mathrm{S}$ (Figura $10 \mathrm{a}$ ), $11^{\circ} \mathrm{S}$ (Figura $10 \mathrm{~b}$ ) y $12.9^{\circ} \mathrm{S}$ (Figura $10 \mathrm{c}$ ), muestra zonas de saturación que oscilan entre el 70 y $90 \%$ de humedad relativa al lado oriental de la Cordillera de los Andes, que se requiere para la formación de nubosidad (Quispe et al., 2003). Aparte de ello, la inestabilidad en la columna atmosférica se observó con los valores negativos de omega que oscilaron entre 0.1 a $3 \mathrm{~Pa} / \mathrm{s}$, siendo el 16 de febrero de 2007 a las 12 UTC donde se evidenció una región con ambiente favorable a la formación de convección profunda sobre los $11^{\circ} \mathrm{S}$. Dadas las condiciones necesarias para el desarrollo de un sistema convectivo sobre Perú, a pesar de las condiciones de inestabilidad en niveles medios, zonas de divergencia en altura y zonas de convergencia en niveles bajos, los niveles bajos en el flujo de humedad y un aumento en la velocidad del viento a $850 \mathrm{hPa}$ transportan la humedad y favorecen su acumulación en la salida del SALLJ.

Además, se muestra la distribución espacial del flujo de humedad especifica (Figura 11 a) en $\mathrm{kg} / \mathrm{kg}$ (línea solida de color azul) a $850 \mathrm{hPa}$, la identificación de un evento SALLJ (Figura 11 b) con altura geopotencial a $200 \mathrm{hPa}$ (mgp, línea solida de color azul) y la precipitación (Figura 11 c) diaria acumulada ( $\mathrm{mm} /$ día, sombreado en colores) superior a $0.1 \mathrm{~mm} /$ día. En general muestra una mayor concentración de la humedad en la salida del chorro, por confluencia de velocidad del viento que favorece la precipitación. Comparación de datos de viento y precipitación

Se observa un mayor grado de asociación entre los valores de $\bar{V}_{m}$ y precipitación diaria acumulada en las horas de la noche 00 UTC (19 hora local), este comportamiento no alcanza valores tan altos en comparación a las 06,12 , y 18 UTC. Se consideraron los valores del viento con los valores negativos debido a su dirección hacia el sur y las precipitaciones acumuladas en 24 horas con valores superiores a los 0.1 $\mathrm{mm} /$ día; es por ello que la relación entre dichas variables es inversamente proporcional. El grado de asociación entre el $\overline{\mathrm{V}}_{\mathrm{m}}$ y precipitación diaria (Figura 12), muestran una relación inversa con mayor concordancia en la zona $2(-0.2$ a -0.6$)$ para las 00,06 y 12 UTC. Es decir, el flujo de vientos debido al forzamiento topográfico influye más en comparación que la zona 1 en todas las horas sinópticas analizadas.

Por otro lado, en zonas planas (zona 1) no se evidencia una asociación entre la precipitación diaria acumulada y la componente del viento meridional del norte, debido a la difluencia de la velocidad del viento que se genera en la zona de entrada del SALLJ y el transporte de la humedad.

Así mismo, los resultados del coeficiente cercanos a 0 (cero) muestran que la precipitación no está solo condicionada por el $\overline{\mathrm{V}}_{\mathrm{m}}$. Cabe señalar que una baja relación entre los flujos de viento y la precipitación, evidencia que no solo depende de un evento SALLJ, sino de la cantidad de humedad disponible en el ambiente, así como las condiciones necesarias para el desarrollo de un complejo convectivo. Además, el flujo de vientos debido al forzamiento topográfico influye más en comparación que la zona 1 en todas las horas sinópticas analizadas.

\section{Conclusiones}

De los resultados obtenidos se evidenció un flujo meridional del norte en niveles de $850 \mathrm{hPa}$ durante el verano austral, siendo febrero el mes que registró mayor número de casos SALLJ al igual que los registrados a las 00 y 12 UTC. Así mismo, el criterio “0” definido por Whiteman (1997) identificó un mayor número de eventos SALLJ en comparación con los identificados utilizando el criterio de Bonner (1968) con una identificación $53.8 \%$ y $46.2 \%$, respectivamente. Así mismo, la zona de influencia con mayor frecuencia se ubica al lado Noreste del territorio peruano, representado por la zona de influencia 1. Sin embargo, los eventos SALLJ identificados no evidencian una alta correlación cuando se asocia a la precipitación debido a la cantidad de casos identificados.

Dada una baja correlación estadística de los eventos SALLJ, estos no son los causantes principales para el desarrollo de las precipitaciones acumuladas en 24 horas, ya que el número de casos SALLJ identificados, en comparación a los datos de precipitación diaria, son muy pocos; por ende, el grado de relación es menor y evidencia que el desarrollo convectivo sobre territorio peruano comprende diversos factores y no solo un flujo de viento en niveles bajos. Y, finalmente, de los casos específicos en los que se analizaron las condiciones sinópticas, se muestra una mayor concentración de humedad en la salida del SALLJ y una convección profunda con altos valores de humedad relativa en el perfil vertical en la salida del SALLJ, un descenso de la altura geopotencial del nivel isobárico de $200 \mathrm{hPa}$ (nivel alto de la troposfera) en la parte central y media del SALLJ, intensifica el flujo meridional hacia el sur en la época del verano austral. 


\section{Agradecimientos}

Los autores agradecen a los profesores del Departamento de Física y Meteorología de la Universidad Nacional Agraria la Molina, por su apoyo en el desarrollo de la investigación.

\section{Literatura citada}

Amador J.A. 2008. The Intra-Americas Sea Low-level Jet: Overview and Future Research. Annals of the New York Academy of Sciences, 1146(1): 153-188. DOI: https://doi.org/10.1196/annals.1446.012.

Bonner W. 1968. Climatology of the low-level jet. Monthly Weather Review, 96(12): 833-849. American Meteorological Society (AMS). https://doi.org/10.1175/15200493(1968)096\%3C0833:COTLLJ\%3E2.0.CO;2.

Celik A.N. 2003. Weibull representative compressed wind speed data for energy and performance calculations of wind energy systems. Energy Conversion and Management, 44(19): 3057-3072. https://doi.org/10.1016/S0196-8904(03)00075-X.

Espinoza J., Ronchail J., Lavado W., Santini W., Vauchel P., Pombosa R., Villacis M., Carranza J., Junquas C., Drapeau G. \& Guyot J. 2011. Las recientes sequías en la cuenca amazónica peruana: Orígenes climáticos e impactos hidrológicos. Revista Peruana Geo-Atmosférica (RPGA), 3: 63-72.

https://web2.senamhi.gob.pe/rpga/pdf/2011_vol03/art5.p df.

Huffman G.J., Adler R.F., Bolvin D.T., Gu G., Nelkin E.J., Bowman K.P., Hong Y., Stocker E.F. \& Wolff D.B. 2007. The TRMM Multisatellite Precipitation Analysis (TMPA): Quasi-Global, Multiyear, Combined-Sensor Precipitation Estimates at Fine Scales. Journal of Hydrometeorology, 8: 38-55. https://citeseerx.ist.psu.edu/viewdoc/download?doi=10.1 $.1 .709 .7651 \&$ rep=rep1\&type $=$ pdf.

Jactel H., Bauhus J., Boberg J., Bonal D., Castagneyrol B., Gardiner B., Gonzalez-Olabarria J.R., Koricheva J., Meurisse N. \& Brockerhoff E.G. 2017. Tree Diversity Drives Forest Stand Resistance to Natural Disturbances. Current Forestry Reports, 3: 223-243. DOI: $10.1007 / \mathrm{s} 40725-017-0064-1$.

Marengo J., Nicolini M., Douglas M. \& Silva P. 2001. Identificación de casos de jets en niveles bajos, del norte (SALLJ) y del sur (SJ) al este de los Andes durante el verano y otoño 1999. Congremet VIII - Climet IX: La meteorología y el medio ambiente en el siglo XXI, Buenos Aires, 7-11 de mayo de 2001. http://mtcm16b.sid.inpe.br/col/cptec.inpe.br/walmeida/2004/08.10 .15.13/doc/Marengo_Identificacion\%20de\%20casos.pdf.

Marengo J., Soares W., Saulo A. \& Nicolini M. 2004. Climatology of the low-level jet east of the Andes as derived from the NCEP-NCAR reanalyses: Characteristics and temporal variability. Journal of Climate, 17(12): 2261-2280. American Meteorological Society. https://doi.org/10.1175/15200442(2004)017\%3C2261:COTLJE\%3E2.0.CO;2.

Quispe N., Avalos G. \& García J. 2003. Complejo Convectivo a Mesoescala caso: Perú 14 de noviembre del 2003. Lima, Perú. Boletín meteorológico. https://hdl.handle.net/20.500.12542/202.
Salio P., Nicolini M. \& Saulo A. 2002. Chaco low level jet characterization during the austral summer season. Journal of Geophysical Research (JGR) Atmospheres, 107(D24): ACL 32-1-ACL 32-17. AGU (Advancing Earth and Space https://doi.org/10.1029/2001JD001315.

Saulo A., Nicolini M. \& Chou C. 2000. Model characterization of the South American low-level flow during the 1997-1998 spring-summer season. Climate Dynamics, 16: 867-881. https://doi.org/10.1007/s003820000085.

Siegel S. \& Castellan J. 1995. Estadística No Paramétrica: Aplicadas a las Ciencias de la Conducta, 282-288. Cuarta edición. Editorial Trillas. México.

Silva (da) B., Alves J., Cavalcanti E. \& Dantas R. 2002. Potencial eólico na direção predominante do vento no Nordeste brasileiro. Revista Brasileira de Engenharia Agrícola e Ambiental, 6(3): 431-439. https://dx.doi.org/10.1590/S1415-43662002000300009.

Sousa A., Jacondino C. \& Paulino E. 2006. Estudo observacional de jatos de baixos níveis ocorridos no litoral norte e nordeste do estado do pará durante os períodos chuvoso (2002) e seco (2003). Revista Brasileira de Meteorologia, 21(2): 170-179.

Torrealba E. \& Amador J. 2010. La corriente en chorro de bajo nivel sobre los Llanos Venezolanos de Sur América. Revista de climatología, 10:1-20. Centro de Investigaciones Geofísicas y Escuela de Física. Costa Rica, San Jose. http://hdl.handle.net/10669/76170.

Touré S. 2005. Investigations on the Eigen-coordinates method for the 2-parameter weibull distribution of wind speed. Renewable Energy, 30(4): 511-521. https://doi.org/10.1016/j.renene.2004.07.007.

Vernekar A., Kirtman B. \& Fennessy M. 2003. Low-Level Jets and Their Effects on the South American Summer Climate as Simulated by the NCEP Eta Model. Journal of Climate, 16(2): 297-311. https://doi.org/10.1175/15200442(2003)016\%3C0297:LLJATE\%3E2.0.CO;2.

Weibull W. 1951. A Statistical Distribution Function of Wide Applicability. Journal of Applied Mechanics, 293-297. The American Society Of Mechanical Engineers. http://web.cecs.pdx.edu/ cgshirl/Documents/WeibullASME-Paper-1951.pdf.

Wilks D. 2006. Statistical Methods in the Atmospheric Sciences. Second edition. International Geophysics [Serie], Volume 100. Elsevier's Science \& Technology. London.

Whiteman C., Bian X. \& Zhong S. 1997. Low level jet climatology from enhanced rawinsonde observations atmosfera a site in the southern great plains. Journal of Applied Meteorology and Climatology, 36(10): 13631376. https://doi.org/10.1175/15200450(1997)036\%3C1363:LLJCFE\%3E2.0.CO;2.

Wu Y. \& Raman S. 1997. Effect of Land-Use Pattern on the Development of Low-Level Jets. Journal of Applied Meteorology and Climatology, 36(5): 573-590. American Meteorological Society. https://doi.org/10.1175/15200450(1997)036\%3C0573:EOLUPO\%3E2.0.CO;2. 
Yupanqui T., Cabrera J. \& Rau P. 2015. Evaluation of TRMM precipitation data for estimation of daily stream flows in small watersheds. 36th IAHR World Congress 28 june -3 july, The Hague / Netherlands. https://www.researchgate.net/profile/Raul-

Tupac/publication/328697644_Evaluation_daily_TRM M_streamflow_Upper_Piura_iahr15/links/5bdc60ad299 bf1124fb539a6/Evaluation-daily-TRMM-streamflowUpper-Piura-iahr15.pdf.
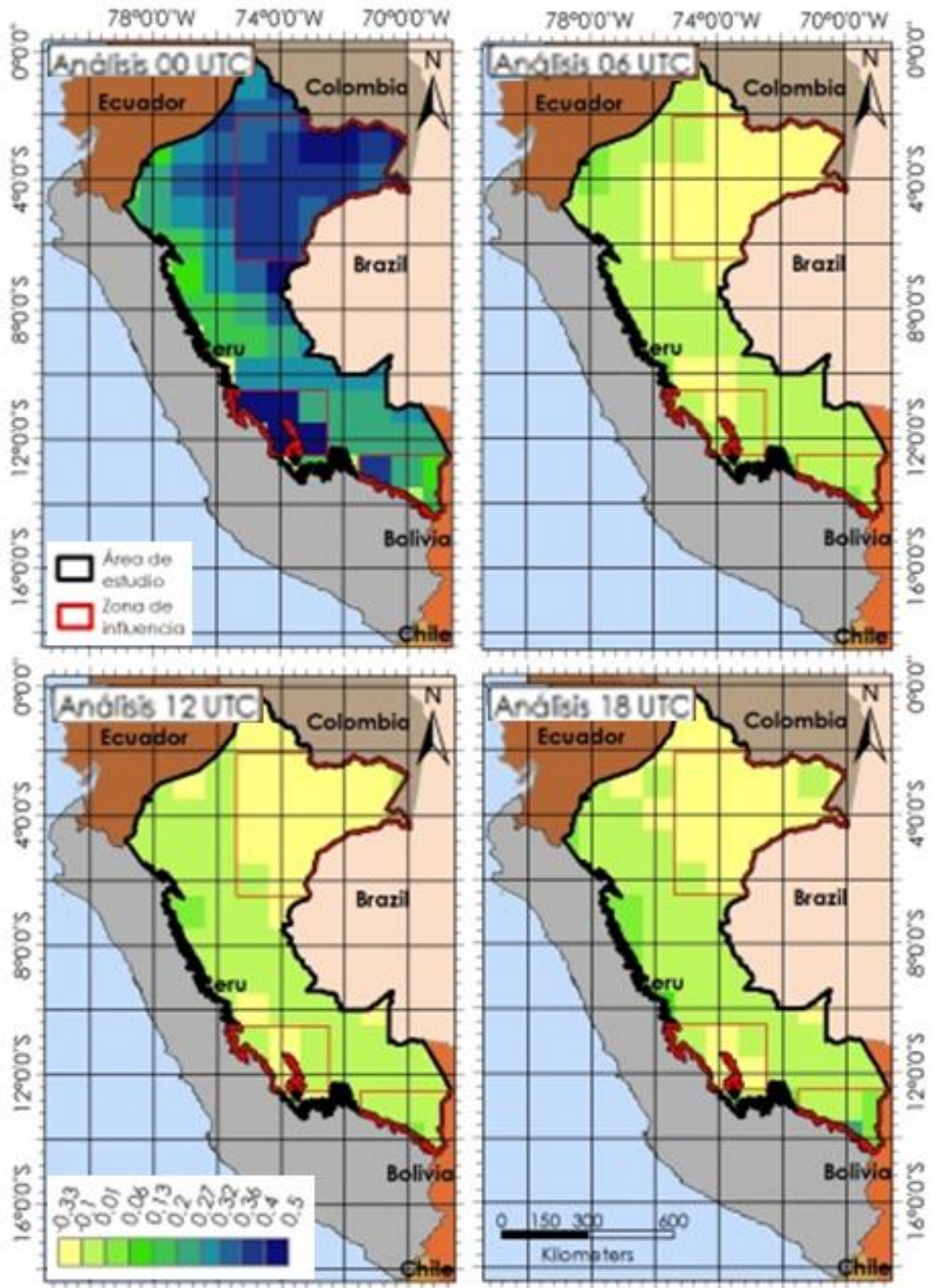

Figura 4. Correlación de viento meridional del norte y cizalladura vertical. 

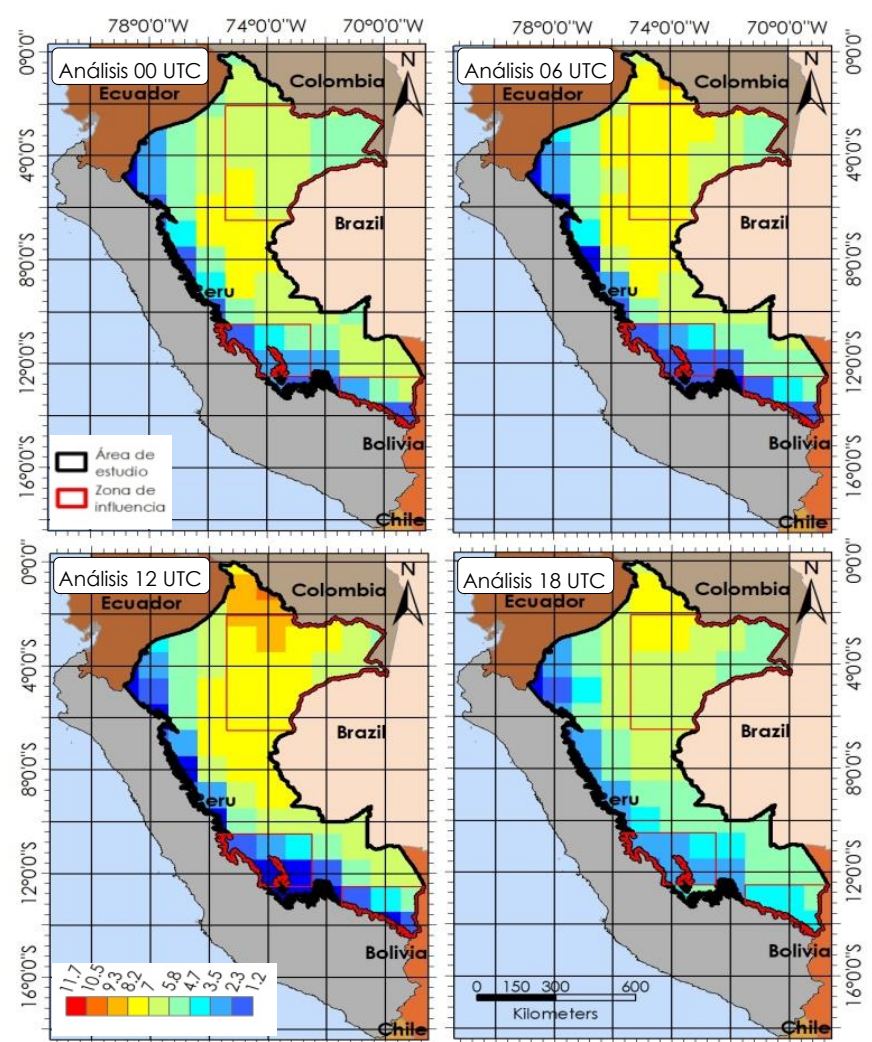

Figura 5. Promedio del viento meridional del norte $(\mathrm{m} / \mathrm{s})$.
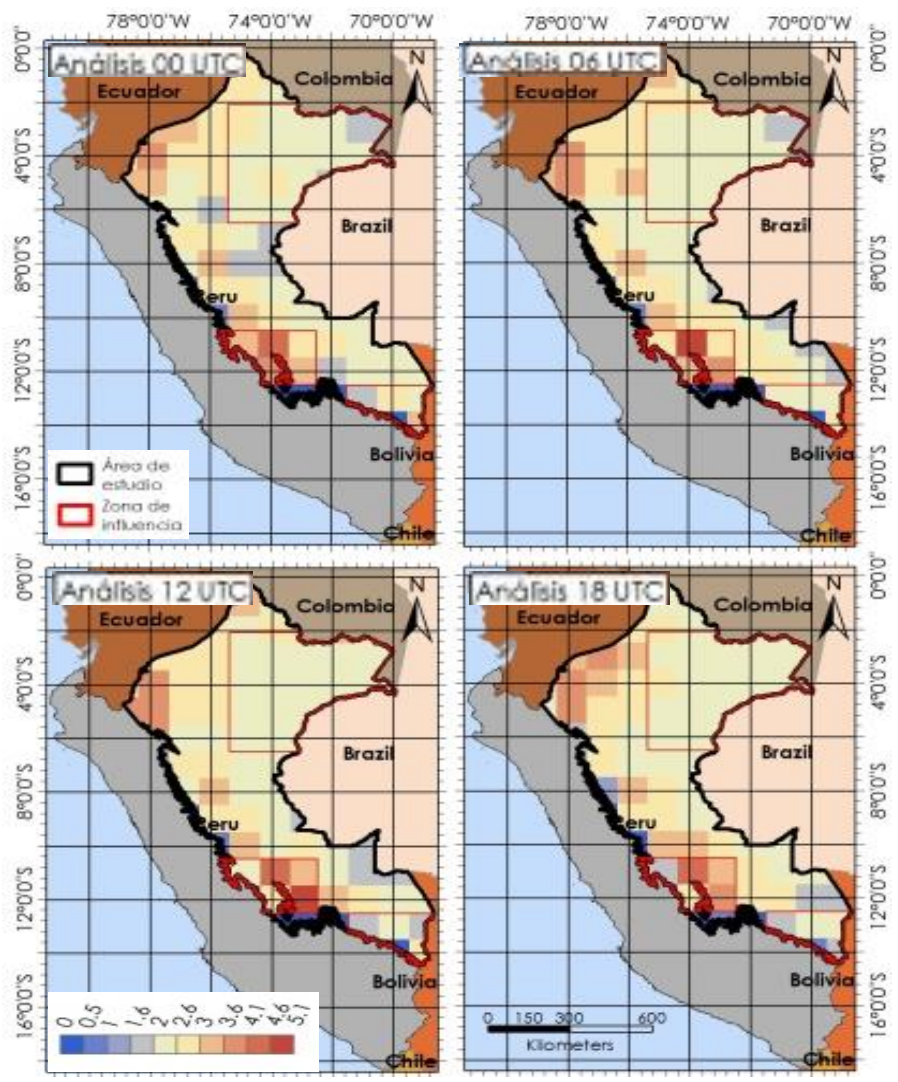

FUENTE: Elaboración propia.

Figura 6. Promedio de cizalladura vertical $(\mathrm{m} / \mathrm{s})$. 
CORRIENTE EN CHORRO DE BAJOS NIVELES Y PRECIPITACIÓN EN LA SELVA DEL PERÚ Julio - Diciembre 2021
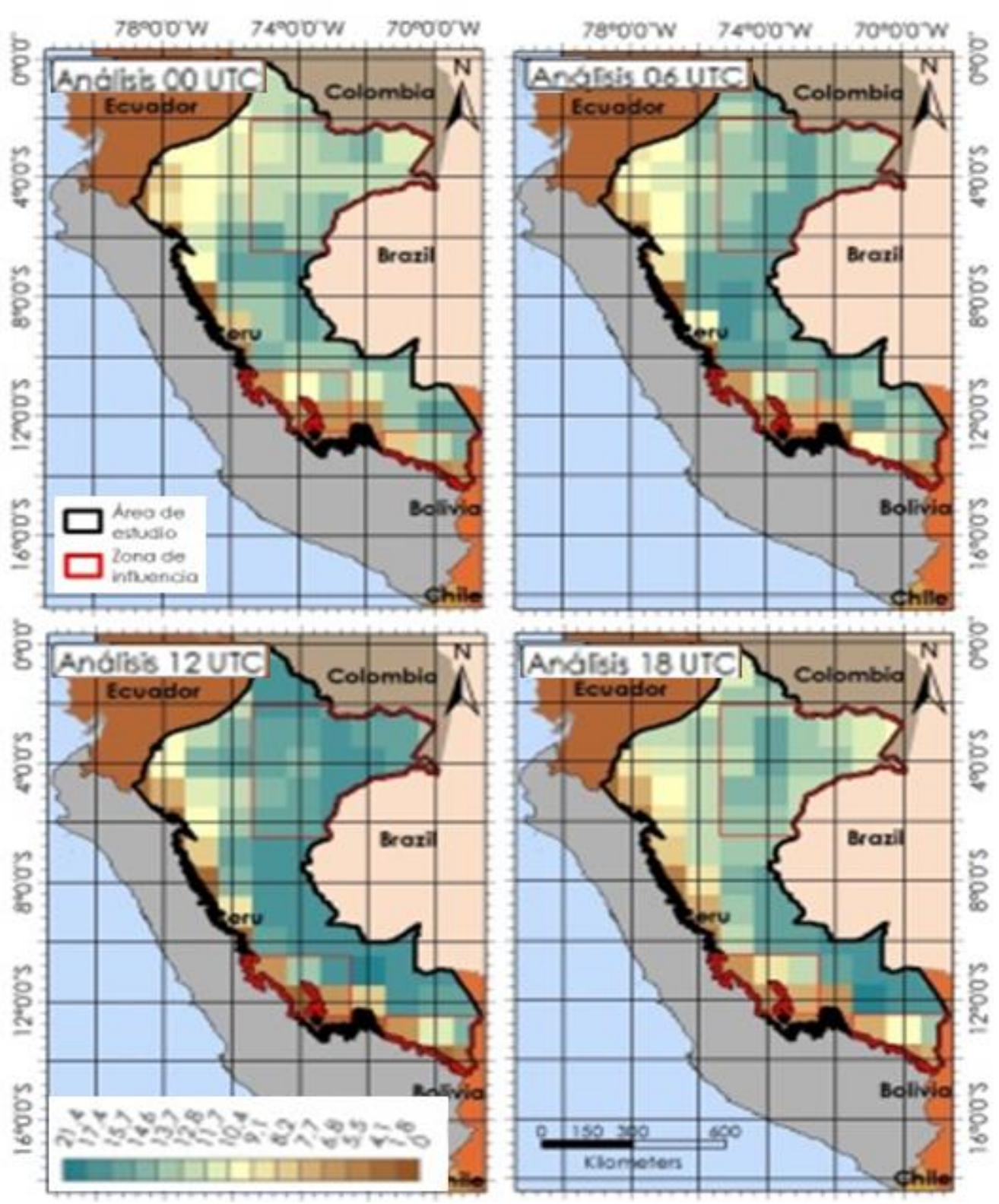

FUENTE: Elaboración propia.

Figura 7. Registro máximo del viento meridional del norte $(\mathrm{m} / \mathrm{s})$. 

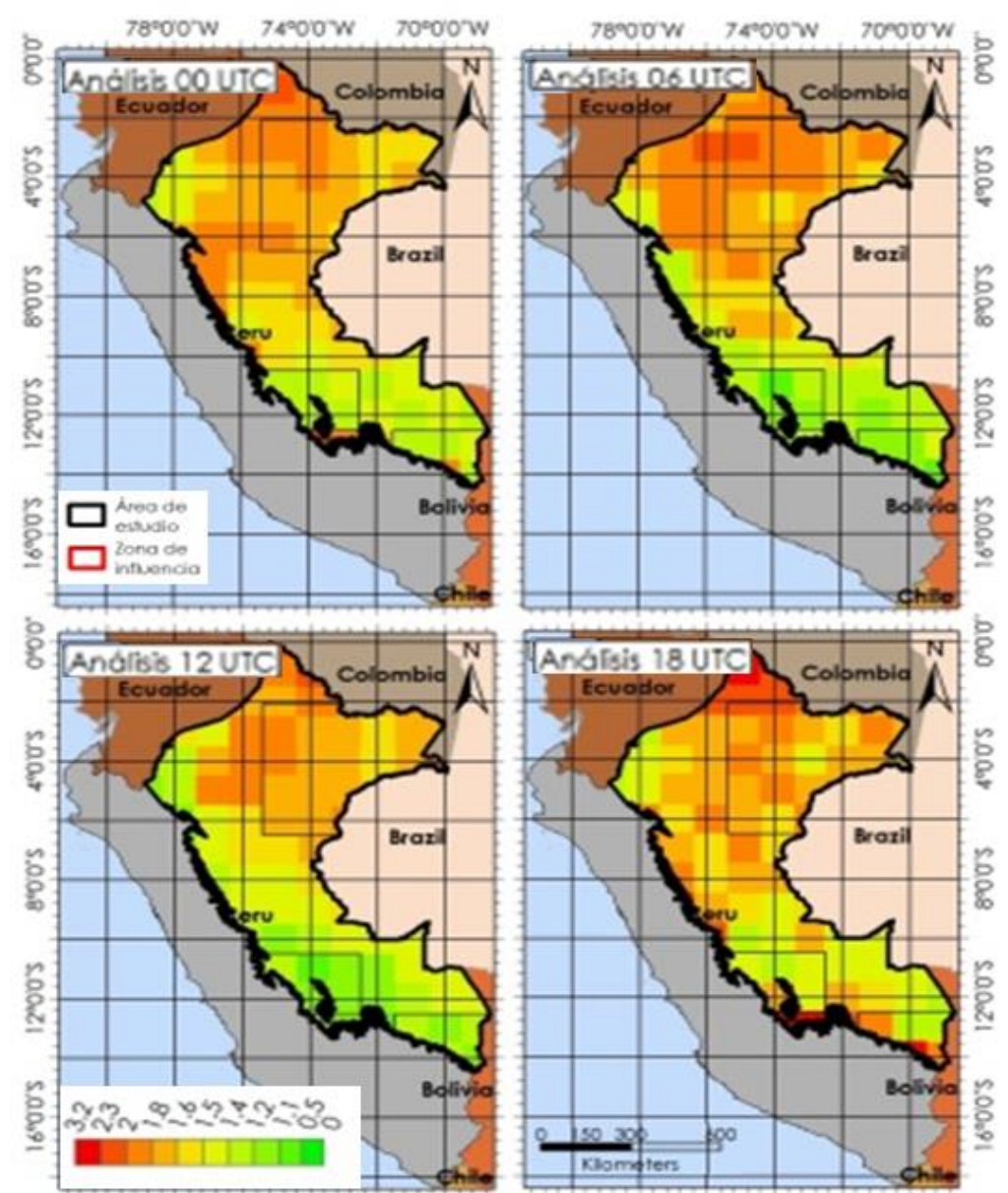

FUENTE: Elaboración propia.

Figura 8. Factor de forma del viento meridional del norte $(\mathrm{m} / \mathrm{s})$.

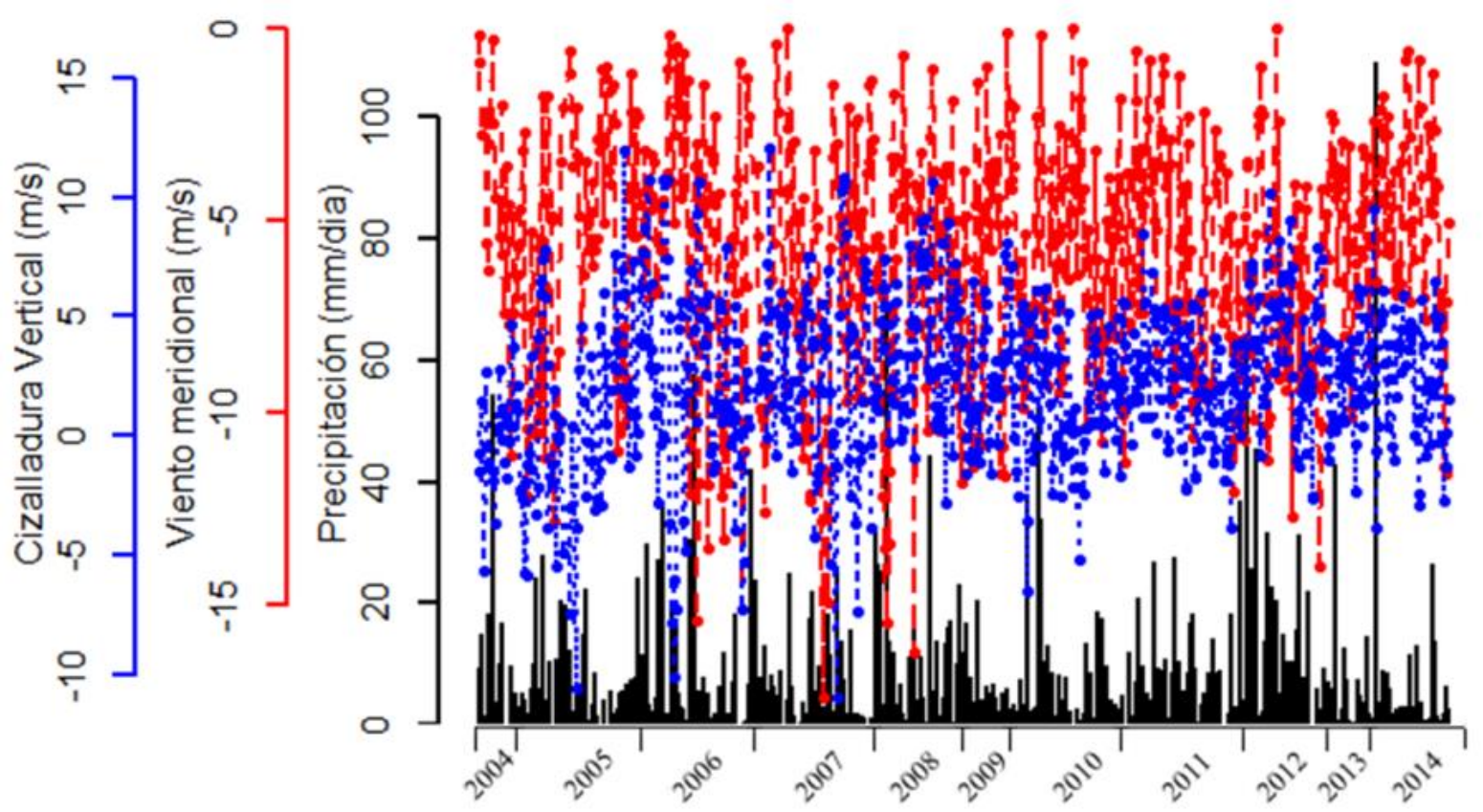

Figura 9. Comportamiento temporal del $\overline{\mathrm{V}}_{\mathrm{m}}$ (líneas rojas), $V_{\text {ciz.vert }}$ (líneas azules) y precipitación (barras) diaria acumulada en m/s y mm/día, respectivamente. Para las 12 UTC, Latitud: $2.9^{\circ} \mathrm{S} /$ Longitud: $72.1^{\circ} \mathrm{W} . \mid$ 

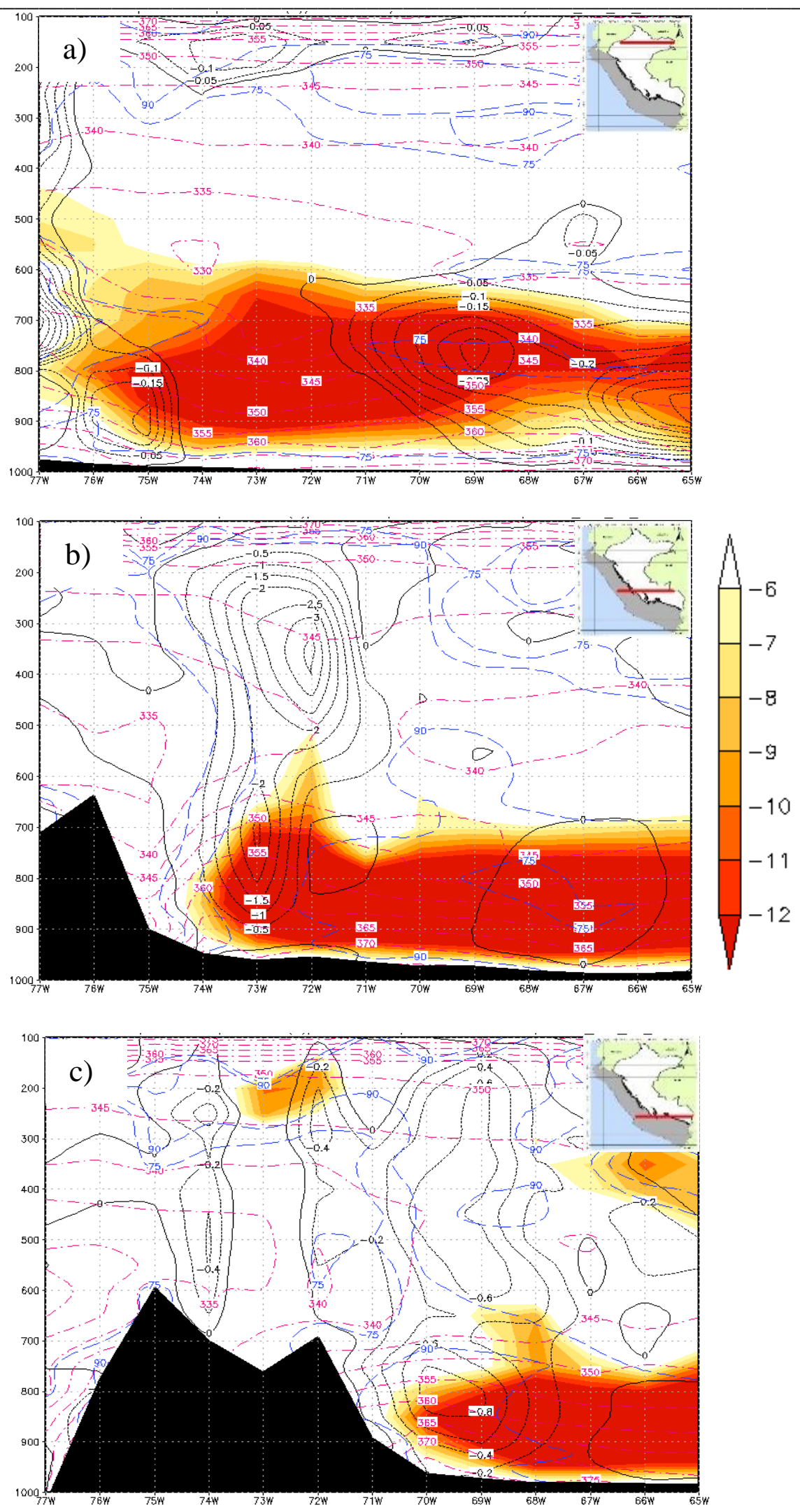

Figura 10. Perfil de viento meridional (m/s, shaded), Omega ( $\mathrm{Pa} / \mathrm{s}$, color negro), Humedad relativa (\%, color azul) y Temp. Potencial equivalente (Kelvin, color cian) del 16 de febrero de 2007 a las 12 UTC. a) Corte latitudinal $2.9^{\circ}$, b) corte latitudinal $11^{\circ} \mathrm{y} \mathrm{c}$ ) corte latitudinal $12.9^{\circ}$. 

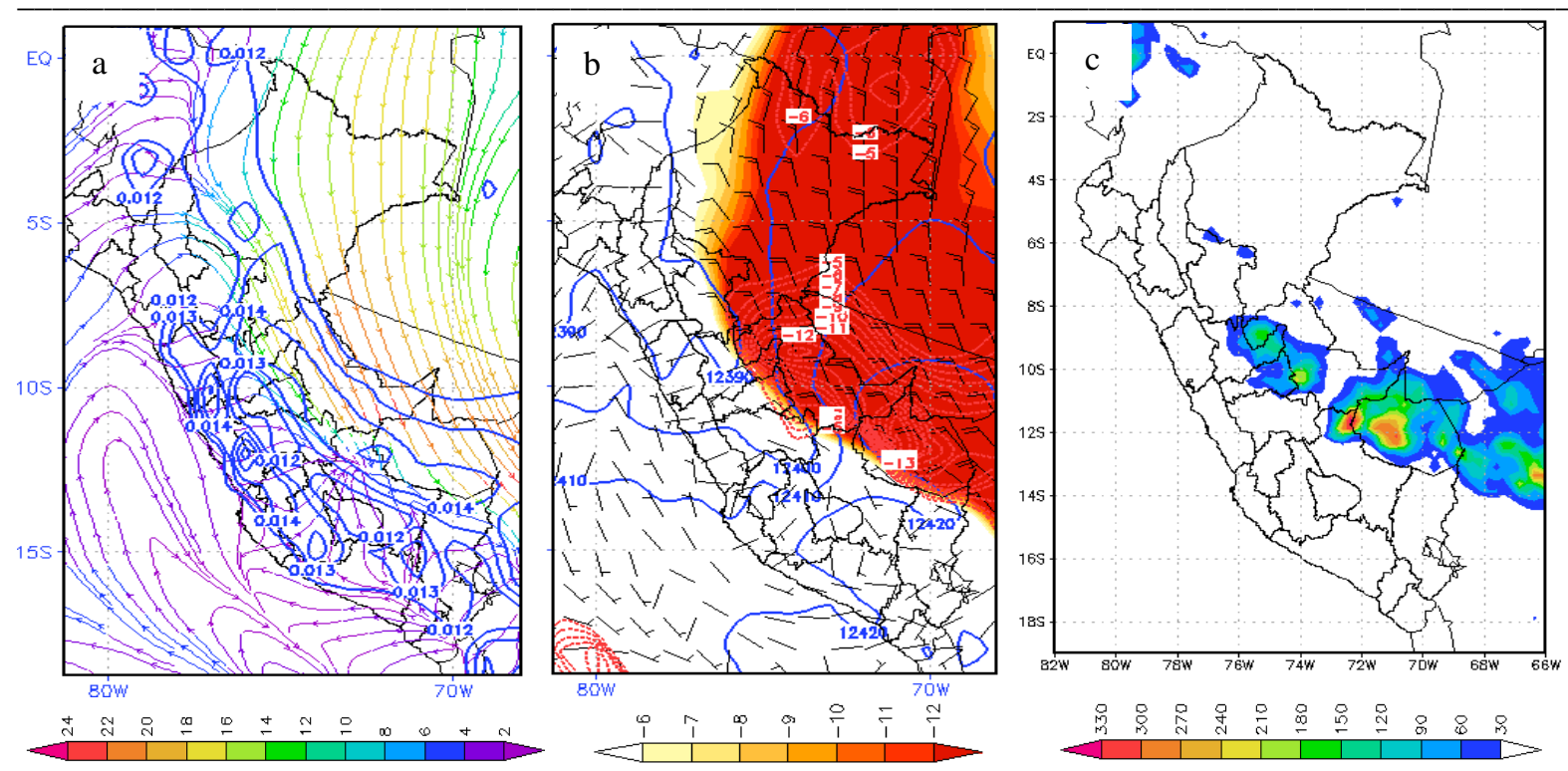

FUENTE: Elaboración propia.

Figura 11.a) Flujo de Humedad especifica (kg/kg, color azul) a 850 hPa, b) Altura geopotencial a 200 hPa (mgp, color azul) y eventos SALLJ, c) Precipitación diaria acumulada (mm/día) / 16 de febrero de 2007 a las 12 UTC.
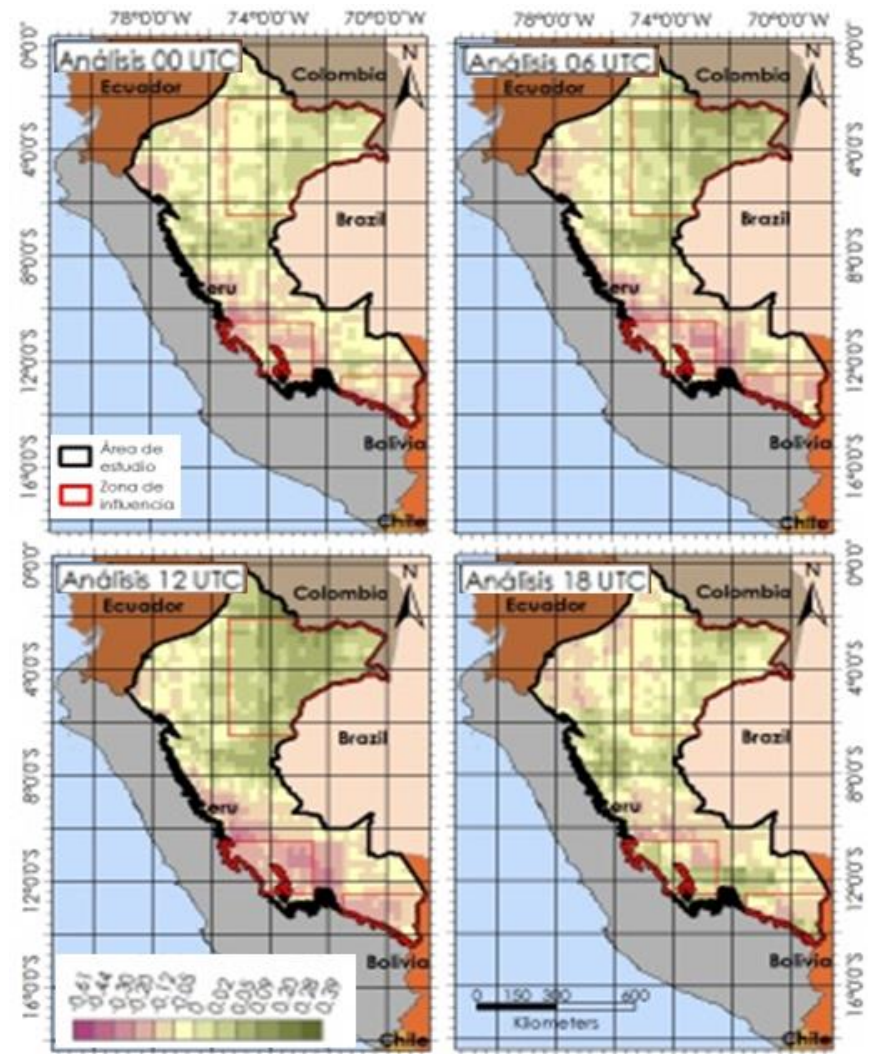

Figura 12. Correlación de viento meridional del norte y precipitación diaria.

\footnotetext{
${ }^{1}$ Comisión Nacional de Investigación y Desarrollo Aeroespacial (CONIDA). jcoello@conida.gob.pe.

${ }^{2}$ Universidad Nacional Agraria la Molina (UNALM). vcmquebec@lamolina.edu.pe.
} 